\title{
A HOMOMORPHISM THEOREM FOR FINITE GRAPHS
}

\author{
MICHAEL ASCHBACHER ${ }^{1}$
}

\begin{abstract}
A homomorphism theorem for finite graphs is established and several group theoretical applications are derived. The proof is taken from a lemma of B. Fischer in his paper classifying groups generated by 3transpositions.
\end{abstract}

In this note a graph is understood to be an ordered pair $\mathcal{G}=(\Omega, \mathcal{E})$ where $\Omega$ is a finite set of vertices and $\mathcal{E}$ is an irreflexive, symmetric relation on $\Omega$. Hence $\mathcal{G}$ is an undirected graph with no loops or multiple edges. For $A$ in $\Omega$ denote by $\mathcal{G}(A)$ the collection of vertices adjacent to $A$ in $\mathcal{G}$. Set $A^{\perp}$ $=\{A\} \cup \mathcal{G}(A)$. For $\alpha \subseteq \Omega$ define

$$
\mathcal{S}(\alpha)=\bigcap_{A \in \alpha} \mathcal{S}(A) .
$$

$\mathcal{G}(\alpha)$ is regarded as a subgraph of $\mathcal{G}$ with adjacency induced from $\mathcal{S} . \mathcal{G}^{c}$ denotes the complementary graph of $\mathcal{G}$, with vertex set $\Omega$ and distinct points adjacent in $\mathcal{G}^{c}$ if they are not adjacent in $\mathcal{G}$. A homomorphism of $\mathcal{G}$ onto a graph $\mathcal{G}^{\prime}$ is a function $x$ from $\Omega$ onto $\Omega^{\prime}$ such that $A$ is adjacent to $B$ if and only if $A x$ is adjacent to $B x$, for all distinct $A x$ and $B x$ in $\Omega^{\prime}$.

Let $\Gamma$ be a partition of $\Omega$. Form the factor graph $\mathcal{G} / \Gamma$ with vertex set $\Gamma$ by joining distinct members $\alpha$ and $\beta$ of $\Gamma$ if some point of $\alpha$ is joined to some point of $\beta$. Then the inclusion map $i$ from $\Omega$ to $\Gamma$ induces a partial homomorphism from $\mathcal{G}$ to $\mathcal{G} / \Gamma$, in that $A$ adjacent to $B$ in $\mathcal{G}$ implies $A i$ is adjacent to $B i$ in $\mathcal{G} / \Gamma$. A contraction of $\mathcal{G}$ is a partition $\Gamma \neq\{\Omega\}$ of $\Omega$ such that the inclusion map is a homomorphism of $\mathcal{G}$ onto $\mathcal{G} / \Gamma$. Partially order the set of contractions of $\mathcal{G}$ by taking $\Gamma_{1}$ less than $\Gamma_{2}$ if each member of $\Gamma_{1}$ is contained in a member of $\Gamma_{2}$. If $\Gamma_{1}$ is less than $\Gamma_{2}$ then $\Gamma_{2}$ corresponds to a contraction of $\mathcal{G} / \Gamma_{1}$, so that there is a natural homomorphism of $\mathcal{G} / \Gamma_{1}$ onto $\mathcal{G} / \Gamma_{2}$. Thus the maximal contractions are of greatest interest.

In this note it is shown that if $\mathcal{G}$ and $\mathcal{G}^{c}$ are connected and $\mathcal{G}$ admits a transitive group of automorphisms then there is a unique maximal contraction of $\mathcal{G}$, and that contraction has nice properties. This result has many applications to finite group theory, several of which are derived within. The proof is taken from Lemma 3.1.1 of B. Fischer's paper classifying groups generated by 3-transpositions [1].

Recall that for $A \in \Omega, \mathcal{S}(A)$ is the subgraph of vertices joined to $A$ in $\mathcal{S} . \mathcal{S}(A)^{c}$ is the complementary graph of $\mathscr{G}(A)$. Let $\mathcal{O}$ be the set of all connected

Received by the editors February 8, 1975 and, in revised form, May 30, 1975.

AMS (MOS) subject classifications (1970). Primary 05C25; Secondary $20 \mathrm{D} 05$.

${ }^{1}$ Partial support supplied by the Alfred P. Sloan Foundation and NSF GP-35678. 
components of $\mathcal{G}(A)^{c}$ as $A$ ranges over $\Omega$. Let $\Re$ be the collection of all maximal elements of $\theta$ under inclusion.

A clump of $\mathcal{G}$ is a proper subset $\alpha$ of $\Omega$ such that $A^{\perp} \subseteq \alpha \cup \mathcal{G}(\alpha)$ for each $A \in \alpha$. Notice that if $\Gamma$ is a contraction of $\mathcal{G}$ and $\alpha \in \Gamma$ then $\alpha$ is a clump of $\mathcal{G}$.

Lemma 1. Let $\pi \in \mathfrak{B}$. Then $\mathcal{S}(\pi)$ is a clump of $\mathcal{S}$, and $\mathcal{S}(\mathcal{S}(\pi))=\pi$.

Proof. Maximality of $\pi$ implies $\pi$ is a connected component of $\mathcal{G}(A)^{c}$.

Lemma 2. Assume $\mathcal{G}$ and $\mathcal{G}^{c}$ are connected, $\pi \in \mathscr{B}, A \in \mathcal{G}(\pi)$, and $\beta$ is a clump of $\mathcal{G}$ containing $A$. Then $\beta \subseteq \mathcal{G}(\pi)$.

Proof. $\pi \subseteq A^{\perp} \subseteq \beta \cup \mathcal{G}(\beta)$ as $\beta$ is a clump.

Since $\pi$ is a connected subgraph of $\dot{\mathcal{G}}^{c}$, if $\pi \cap \mathcal{G}(\beta)$ is nonempty then $\pi \subseteq \mathcal{G}(\beta)$ and hence $\beta \subseteq \mathcal{G}(\pi)$. So we may take $\pi \subseteq \beta . \beta \neq \Omega$ and $\mathcal{G}$ is connected, so there exists $B \in \mathcal{G}(\beta)$. By Lemma $1, \beta \subseteq B^{\perp} \subseteq \pi \cup \mathcal{G}(\pi)$. $\pi \subseteq \beta$ so $\mathcal{S}(\beta) \subseteq \mathcal{G}(\pi)$. Hence as $\beta$ is a clump, $C^{\perp} \subseteq \beta \cup \mathcal{G}(\beta) \subseteq \pi \cup \mathcal{G}(\pi)$ for each $C \in \pi$. By Lemma $1, C^{\perp} \subseteq \pi \cup \mathcal{G}(\pi)$ for each $C \in \mathcal{G}(\pi)$. So $\pi \cup \mathscr{S}(\pi)$ contains a connected component of $\mathcal{S}$ and hence $\Omega=\pi \cup \mathcal{S}(\pi)$. But then $\mathcal{G}^{c}$ is not connected, a contradiction.

We define a collection $\Gamma(\mathcal{G})$ of subsets of $\Omega$ :

$$
\Gamma(\mathcal{G})=\{\mathcal{G}(\pi): \pi \in \mathscr{B}\} \cup\left\{\{A\}: A \in \Omega-\bigcup_{\pi \in \mathscr{B}} \mathcal{S}(\pi)\right\} .
$$

Lemma 3. Assume $\mathcal{G}$ and $\mathcal{G}^{c}$ are connected. Then $\Gamma(\mathcal{G})$ is a contraction.

Proof. Lemmas 1 and 2.

THEOREM 4. Let $\mathcal{G}$ and $\mathcal{G}^{c}$ be connected with $G=\operatorname{Aut}(\mathcal{G})$ transitive on $\Omega$. Then

(1) $\Gamma(\mathcal{G})$ is the unique maximal contraction of $\mathcal{G}$.

(2) $\Gamma(\mathcal{S})$ is a system of imprimitivity for the action of $G$ on $\Omega$.

(3) Let $\alpha \in \Gamma(\mathcal{G})$. Then $\mathcal{G}(\alpha)^{c}$ and $\left(\mathcal{G}^{c}(\alpha)\right)^{c}$ are connected subgraphs of $\mathcal{G}^{c}$ and $\mathcal{G}$, respectively.

Proof. (1) follows from Lemmas 2 and 3. (1) implies (2). By definition of $\Gamma(\mathcal{G}), \mathcal{S}(\alpha)^{c}$ is connected. Contractions of $\mathcal{G}$ are contractions of $\mathcal{G}^{c}$. Hence by symmetry $\left(\mathcal{G}^{c}(\alpha)\right)^{c}$ is connected.

In the remainder of this note let $G$ be a finite group and $\Omega$ a conjugacy class of subgroups of $G$ generating $G$. Define $\mathcal{G}$ to be the graph with vertex set $\Omega$ and $A$ joined to $B$ if $A \neq B$ and $[A, B]=1$ (i.e. $A$ and $B$ commute). Then $G$ acts as a transitive group of automorphisms of $\mathcal{G}$. As $G$ is generated by and is transitive on $\Omega, \mathcal{G}^{c}$ is connected.

LEMma 5. Assume $\mathcal{G}$ is connected and for $A \in \Omega$, distinct orbits on $\mathcal{S}(A)$ of the group $\langle\mathcal{S}(A)\rangle$ generated by $\mathcal{S}(A)$ commute. Let $A \in \alpha \in \Gamma(\mathcal{S})$ and $\pi=\mathcal{G}(A)$ $-\alpha$. Then

(1) $\langle\pi\rangle$ is transitive on $\pi$.

(2) Let $A^{g} \in \pi$. Then $G=\left\langle\pi, \pi^{g}\right\rangle$.

(3) $\alpha$ is a set of imprimitivity for the action of $G$ on $\Omega$ and $\pi$ is a union of conjugates of $\alpha$. 
(4) $\Omega-(\alpha \cup \pi)$ is a connected subgraph of $\mathcal{G}$.

Proof. Parts (3) and (4) are a restatement of Theorem 4 in the context of this example. Also by Theorem $4, \pi$ is a connected subgraph of $\mathcal{G}^{c}$, so as distinct orbits of $\langle\mathscr{S}(A)\rangle$ on $\mathscr{S}(A)$ commute, $\langle\pi\rangle$ is transitive on $\pi$. Let $\Gamma$ be the set of points conjugate to $A$ or $A^{g}$ under $\left\langle\pi, \pi^{g}\right\rangle$. $\mathscr{S}(A) \subseteq \alpha \cup \pi \subseteq \pi \cup \pi^{g}$ $\subseteq \Gamma$ by (1). Similarly $\mathcal{G}\left(A^{g}\right) \subseteq \Gamma$. Thus $\Gamma$ contains a connected component of $\mathcal{G}$, so as $\mathcal{G}$ is connected, (2) holds.

We conclude with an example illustrating how Lemma 5 can be used. $\Omega$ is a class of 3-transpositions of $G$ if $\Omega$ is a conjugacy class of involutions such that $|a b| \leq 3$ for each $a, b \in \Omega$. We derive Fischer's fundamental theorem on the permutation action of $G$ on a class $\Omega$ of 3-transpositions.

THEOREM (FISCHER). Let $\Omega$ be a conjugacy class of 3-transpositions of a finite group $G$. Assume the commutator group $G^{\prime}$ of $G$ is simple. Then $G$ acts by conjugation as a rank 3 permutation group on $\Omega$.

Proof. First some remarks.

(1) $\mathcal{G}$ and $\mathcal{G}^{c}$ have edges.

(2) If $a \in \Omega, b, c \in \mathcal{G}^{c}(a)$, and $c \in \mathcal{G}(b)$ then $\langle a, b, c\rangle \cong S_{4}$.

By Glauberman's $Z^{*}$-theorem $\mathcal{G}$ has an edge. As $G^{\prime}$ is simple, $\mathcal{G}^{c}$ has an edge. (2) is well known. See for example 1.4 in [1]. Next we claim

(3) $G$ is primitive on $\Omega$.

Assume not and let $\alpha^{G}$ be a system of imprimitivity, let $b$ and $c$ be distinct points of $\alpha$, and $a \in \beta=\alpha^{g} \neq \alpha$. We show $b c$ lies in the kernel $K$ of the action of $G$ on $\alpha^{G}$. As $G^{\prime}$ is simple, $G^{\prime} \leq K$, so $G^{\prime}=G^{\prime}\langle b\rangle$ acts on $\alpha$, a contradiction. So assume $\beta^{b c} \neq \beta$. Notice

(4) $\alpha$ is a clump.

For if $a \in \mathcal{G}(b)$ then $\langle a, c\rangle$ acts on $\alpha$ so $a$ is not conjugate to $c$ in $\langle a, c\rangle$ and hence by the 3 -transposition property, $a \in \mathcal{S}(c)$. In particular as $\beta^{b c} \neq \beta$, (4) says $a b$ and $a c$ have order 3 . If $b \in \mathcal{G}(c)$ then by $(2),\{b, c\}$ is conjugate to $\left\{a, a^{b c}\right\}$ in $\langle a, b, c\rangle$, so $a^{b c} \in \beta$, a contradiction. Thus $b c$ has order 3 for each $c$ in $\alpha-\{b\}$. So by (4),

$$
\alpha \subseteq W_{b}=\{c \in \Omega: \mathcal{G}(b)=\mathcal{G}(c)\}
$$

and as $\left(W_{b}\right)^{G}$ is a system of imprimitivity we may take $\alpha=W_{b}$. So as $a b$ has order $3, \beta^{b}=\left(W_{a}\right)^{b}=W_{a b}=W_{b a}=\left(W_{b}\right)^{a}=\alpha^{a}$. Similarly $\beta^{c}=\alpha^{a}$. So $\beta^{b c}$ $=\beta$, and (3) is finally established.

As the collection of connected components of $\mathcal{G}$ is a system of imprimitivity, (1) and (3) imply $\mathcal{G}$ is connected. Now by Lemma 5 , the stabilizer $G_{a}$ of $a \in \Omega$ is transitive on $\mathcal{G}(a)$. Let $b, c \in \mathcal{G}^{c}(a)$, with $b \in \mathcal{S}(c)$. By (2), $b^{a d}=c$, where $\{d\}=\mathcal{G}(a) \cap\langle a, b, c\rangle$. In particular by Lemma 5 , part (4), $\left\langle a^{\perp}\right\rangle \leq G_{a}$ is transitive on $\mathcal{S}^{c}(a)$. So $G_{a}$ has orbits $\{a\}, \mathcal{G}(a)$, and $\mathcal{S}^{c}(a)$ on $\Omega$.

\section{REFERENCE}

1. B. Fischer, Finite groups generated by 3-transpositions. I, Invent. Math. 13 (1971), 232-246. MR 45 \#3557.

Department of Mathematics, California Institute of Technology, Pasadena, CaliforNIA 91125 